EPJ Web of Conferences 71, 00096 (2014)

DOI: 10.1051/epjconf/20147100096

(C) Owned by the authors, published by EDP Sciences, 2014

\title{
The 3D structure of the hadrons: recents results and experimental program at Jefferson Lab
}

\author{
C. Muñoz Camacho ${ }^{1, a}$ \\ ${ }^{1}$ Institut de Physique Nucléaire, Orsay (France)
}

\begin{abstract}
The understanding of Quantum Chromodynamics (QCD) at large distances still remains one of the main outstanding problems of nuclear physics. Studying the internal structure of hadrons provides a way to probe QCD in the non-perturbative domain and can help us unravel the internal structure of the most elementary blocks of matter. Jefferson Lab (JLab) has already delivered results on how elementary quarks and gluons create nucleon structure and properties. The upgrade of JLab to $12 \mathrm{GeV}$ will allow the full exploration of the valence-quark structure of nucleons and the extraction of real threedimensional pictures. I will present recent results and review the future experimental program at JLab.
\end{abstract}

\section{Introduction}

Quantum Chromodynamics (QCD), the theory of strong interactions, presents unique features compared to its counterpart for electromagnetic interactions, Quantum Electrodynamics (QED). Firstly, in contrast to QED, in QCD we cannot observe the elementary degrees of freedom of the theory (quarks and gluons). This characteristic is usually known as confinement. A second key property of QCD is its so-called asymptotic freedom: the effective coupling constant $\alpha_{s}$ becomes very small $(<1)$ at small distances $(\lesssim 0.2 \mathrm{fm})$. On the other hand, at the scale typical of hadron formation, $\alpha_{s}$ is large and does not allow perturbative calculations, as it is customary in QED.

Studying the internal structure of the nucleon (protons and neutrons) is one of the best ways to shed light on the workings of QCD. Indeed, the nucleon is one of the simplest particles bound by the strong force, yet showing an extreme theoretical complexity. It is a relativistic, strongly coupled, quantum many-body problem that presents extreme challenges to theory. Strong efforts have been devoted in the last decades to numerical calculations by discretizing the QCD lagrangian in a lattice [1]. Also, effective field theories have been developed in some limits (large number of colors, small mass of quarks... ) [2].

However, we will concentrate here in the efforts carried out to study the structure of the nucleon experimentally, and in particular through high energy scattering of leptons. Many fundamental questions about the nucleon still remain unanswered, such us the origin of its mass or its spin. The mass of quarks only account for a very small fraction of the nucleon mass, and most of it seems to come from the motion of quarks and gluons inside.

a e-mail: munoz@ipno.in2p3.fr

This is an Open Access article distributed under the terms of the Creative Commons Attribution License 2.0, which permits unrestricted use, distribution, and reproduction in any medium, provided the original work is properly cited. 


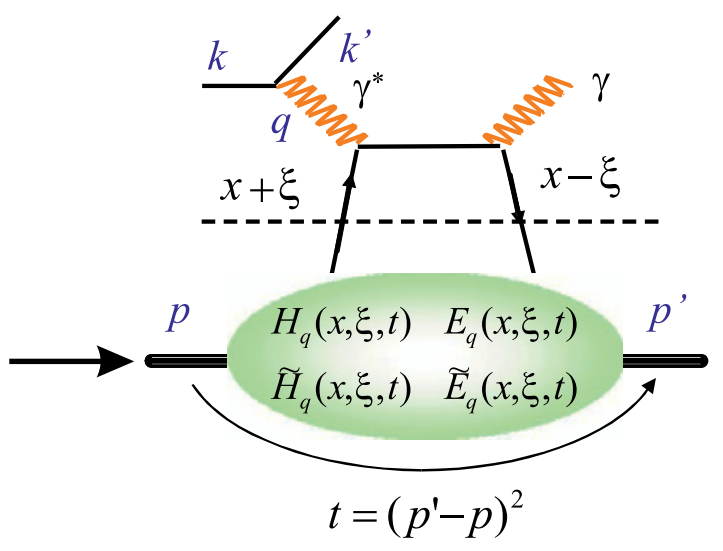

Figure 1. Handbag diagram for DVCS. At sufficiently high virtuality of the initial photon, the interaction occurs with one single quark of the nucleon, which absorbs the virtual photon and radiates a high energy photon in the final state. There is a finite momentum transfer $t$ to the nucleon, whereas the quark itself changes its longitudinal momentum fraction from $x+\xi$ to $x-\xi$. The amplitude of the process factorizes into a hard perturbative part (top) and a soft term parametrized by 4 GPDs (bottom).

Traditionally, nucleon structure has been studied in two complementary ways. Through elastic scattering, measurements of form factors can be related to the charge and magnetization distributions inside the nucleon. This provides a distribution of its constituents in position space. On the other hand, through Deeply Inelastic Scattering (DIS) we can relate parton distributions functions (PDFs) to the momentum distribution of quarks and gluons. They are complementary ways with similar drawbacks. Form factors do not provide any information about the dynamics of the system, whereas parton distributions do not tell us anything about the position of the constituents. A more complete picture can be gathered from the correlation between position and momentum spaces. These correlations are accessible through a new kind of functions that generalize PDFs and are therefore known as Generalized Parton Distributions (GPDs) [3-5].

These new functions are accessible experimentally through exclusive processes in DIS kinematics. The simplest process of this type is Deeply Virtual Compton Scattering (DVCS): $\gamma^{*} p \rightarrow \gamma p$, usually realized using electroproduction of real photons $(e p \rightarrow e p \gamma)$.

\section{Deeply virtual Compton scattering}

In the Bjorken limit of high virtuality $Q^{2}=-q^{2}$ and high energy $v$ of the initial photon, but fixed $x_{B}=Q^{2} / 2 M v$ ( $M$ being the nucleon mass), the DVCS process is represented by the scattering off a single quark of the nucleon (handbag diagram shown in figure 1). The amplitude of the process factorizes into a hard component, calculable using perturbative QCD, and a soft part that can be parametrized by 4 GPDs. GPDs depend on 3 variables: $x$ (the average momentum of the interacting quark), $\xi$ (half the quark momentum difference between the final and initial states) and $t=\left(p^{\prime}-p\right)^{2}$ (the overall momentum transfer to the nucleon).

GPDs reduce to ordinary parton distributions for $\xi=0$ and $t=0$, and their first moments are the form factors measured in elastic scattering (for example $\int d x H^{q}(x, \xi, t)=F_{1}^{q}(t)$, for each flavor of quark $q$ ). However, they contain new information on the nucleon internal structure. In particular, they provide access to the contribution of the angular momentum of quarks $J$ to the nucleon spin, through Ji's sum rule [5]:

$$
J=\frac{1}{2} \int_{-1}^{1} d x x[H(x, \xi, 0)+E(x, \xi, 0)] .
$$

Experimentally, DVCS interferes with the so-called Bethe-Heitler $(\mathrm{BH})$ process (figure 2) where the photon is emitted by the initial or final electron, instead of the proton. The BH process can be calculated exactly in QED as a function of the nucleon form factors $F_{1}$ and $F_{2}$. We can use the 


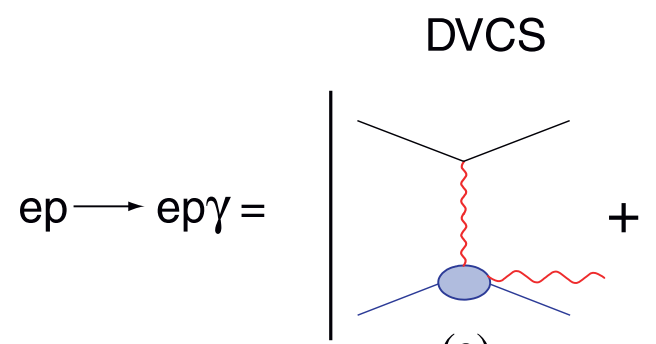

(a)

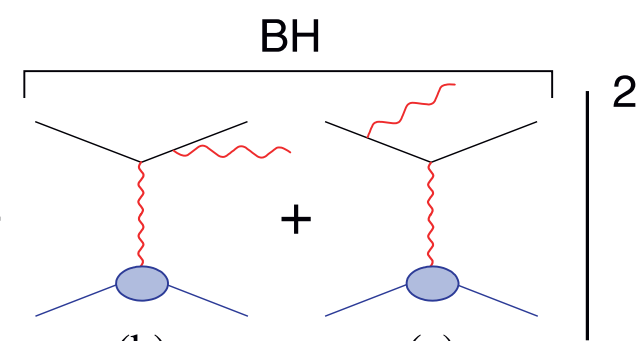

(b) (c)

Figure 2. Interference between DVCS (a) and the Bethe-Heitler (BH) process (b and c), where the photon is emitted by the initial or final electron, instead of the nucleon.

interference of DVCS with a known process to our advantage by using a polarized electron beam. Indeed, at leading order in $1 / Q$ (leading twist), the difference of the photon electroproduction cross sections with opposite beam helicities is proportional to the imaginary part of the interference term. On the other hand, the unpolarized cross section depends only on the real part of the interference term, and the $|\mathrm{DVCS}|^{2}$ contribution. To wit:

$$
\begin{array}{cccc}
d^{5} \vec{\sigma}-d^{5} \stackrel{\leftarrow}{\sigma} & \propto & \mathfrak{J} m\left(T^{B H} \cdot T^{D V C S}\right) \\
d^{5} \vec{\sigma}+d^{5} \stackrel{\leftarrow}{\sigma} & \propto & |B H|^{2}+\mathfrak{R} e\left(T^{B H} \cdot T^{D V C S}\right)+|D V C S|^{2}
\end{array}
$$

This is particularly interesting because GPDs appear differently in the real and the imaginary part of the DVCS amplitude:

$$
T^{D V C S}=\int d x \frac{H(x, \xi, t)}{x-\xi+i \epsilon}+\cdots=\mathcal{P} \int d x \frac{H(x, \xi, t)}{x-\xi}-i \pi H(x=\xi, \xi, t)+\ldots
$$

While the real part is related to the integral of the GPD over $x$, the imaginary part accesses the GPD itself, but along a particular kinematic line $x=\xi$.

\section{Experimental program at Jefferson Lab}

Constraining GPDs through experiments is an ambitious project. There are 4 GPDs for the nucleon and each of them depend on 3 distinct kinematic variables. However, by using different combinations of beam and target polarizations, we can tune our sensitivity to one or several of the GPDs in particular. For example:

$$
\begin{array}{rc}
d \sigma_{L U} \propto & \sin \phi \cdot \mathcal{I} m\left\{F_{1} \mathcal{H}+x_{B}\left(F_{1}+F_{2}\right) \tilde{\mathcal{H}}-\left(t / 4 M^{2}\right) F_{2} \mathcal{E}\right\} d \phi \\
d \sigma_{U L} \propto & \sin \phi \cdot \operatorname{Im} m\left\{F_{1} \tilde{\mathcal{H}}+x_{B}\left(F_{1}+F_{2}\right)\left(\tilde{\mathcal{H}}+x_{B} / 2 \mathcal{E}\right)-x_{B}\left(t / 4 M^{2}\right) F_{2} \tilde{\mathcal{E}} \ldots\right\} d \phi \\
d \sigma_{L L} \propto & (A+B \cos \phi) \cdot \mathcal{R}\left\{\left\{F_{1} \tilde{\mathcal{H}}+x_{B}\left(F_{1}+F_{2}\right)\left(\tilde{\mathcal{H}}+x_{B} / 2 \mathcal{E}\right) \ldots\right\} d \phi\right. \\
d \sigma_{U T} \propto & \cos \phi \cdot \operatorname{Im} m\left\{\left(t / 4 M^{2}\right)\left(F_{2} \mathcal{H}-F_{1} \mathcal{E}\right)+\ldots\right\} d \phi,
\end{array}
$$

where the first and second subscripts of $d \sigma$ indicate the polarization of the beam and the target respectively (U: unpolarized, L: longitudinally polarized, T: transversally polarized). Therefore, as many combinations of observables as possible are needed in order to better pin down the different GPDs. Also, a wide kinematic coverage is necessary. Figure 3 shows the $Q^{2}$ and $x_{B}$ coverage for 


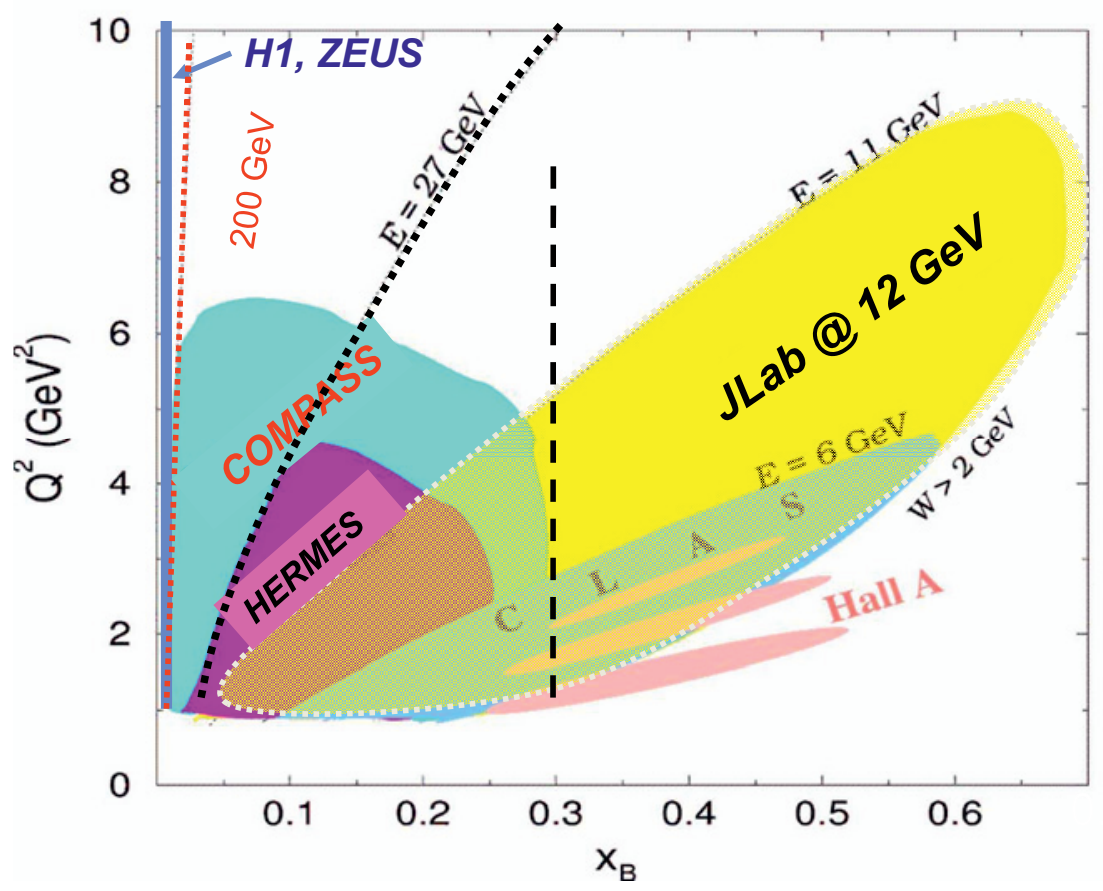

Figure 3. Kinematic complementarity between different facilities. The valence region is almost exclusively covered by Jefferson Lab and its upgrade to $12 \mathrm{GeV}$, while overlapping significantly with kinematics measured by HERMES at DESY.

GPD-related measurements in different facilities worldwide. The valence region is basically covered by Jefferson Lab and will be greatly expanded by its upgrade to $12 \mathrm{GeV}$. The Jefferson Lab program will be the focus of the following sections.

The HERMES experiment at DESY has a wealth of data on exclusive reactions. Also, the COMPASS experiment at CERN is planning to perform measurements on GPDs. These two programs will not be covered herein.

\section{JLab results on DVCS}

Jefferson Lab provides a $6 \mathrm{GeV}$ longitudinally polarized (> 85\%) continuous electron beam of high intensity $(>100 \mu \mathrm{A})$, reaching luminosities of up to $10^{38} \mathrm{~s}^{-1} \mathrm{~cm}^{-2}$. Three experimental Halls (A, B and C) with fixed targets and dedicated detectors can take data independently. An upgrade of its beam energy to $12 \mathrm{GeV}$ is currently underway and will be available during the present year 2014 .

\subsection{Hall A program}

The GPD program with deep exclusive reactions in Hall A is devoted to high precision tests of the $Q^{2}$ dependence of cross sections. Indeed, GPD measurements at Jefferson Lab rely on the assumption that deep exclusive reactions are well described by their leading twist mechanism. Theoretically this is true at high $Q^{2}$. The value of $Q^{2}$ at which this approximation is valid experimentally needs to be 

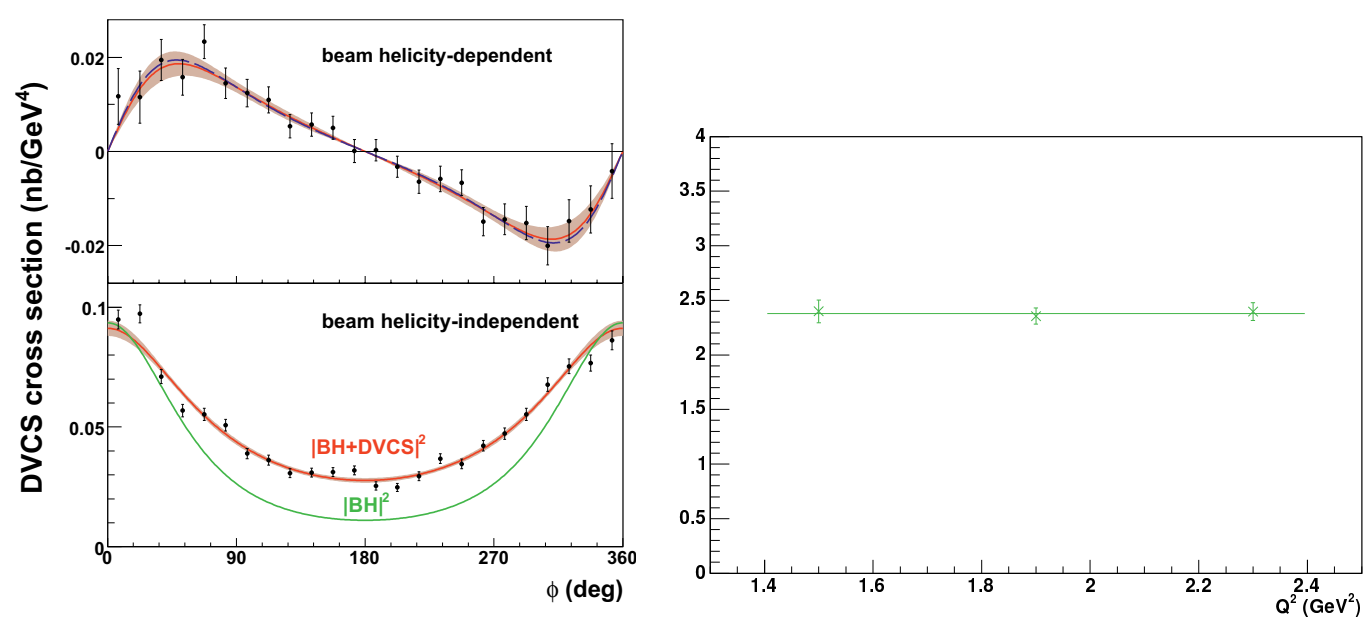

Figure 4. Left: Helicity-dependent (top) and helicity-independent (bottom) photon electroproduction cross section for $Q^{2}=2.3 \mathrm{GeV}^{2}, x_{B}=0.36$ and $t=-0.28 \mathrm{GeV}^{2}$, as a function of the angle $\phi$ between the leptonic and hadronic planes. Right: linear combinations of GPDs $\operatorname{Im} C^{I}$ (Eq. 9) as a function of $Q^{2}$.

determined and the contributions of higher twist components to observables need to be quantified. The $Q^{2}$ dependence of cross sections is the only unambiguous way to separate higher twist contributions to DVCS and other exclusive channels.

Figure 4 (left) shows the helicity-dependent and helicity-independent cross sections for one of the bins in $Q^{2}$ and $t$ measured in Hall A [6], as a function of the angle $\phi$ between the leptonic and the hadronic planes. The $Q^{2}$ dependence of the imaginary part of the linear combinations of GPDs of Eq. (5) is presented in figure 4 (right) :

$$
\operatorname{Im} C^{I}=\operatorname{Im}\left\{F_{1} \mathcal{H}+x_{B}\left(F_{1}+F_{2}\right) \tilde{\mathcal{H}}-\left(t / 4 M^{2}\right) F_{2} \mathcal{E}\right\} .
$$

As shown, within the limited $Q^{2}$ range of the experiment, the amplitude of the $\sin \phi$ contribution to $\sigma_{L U}$ is independent of $Q^{2}$. This suggests that the DVCS process is well represented by the handbag diagram of figure 1. A second important result is that the total cross section (DVCS+BH) deviates greatly from the BH contribution alone. This means that both the $\mathrm{DVCS}^{2}$ and the BH.DVCS interference term contribute significantly to the unpolarized cross section. Unfortunately, an azimuthal analysis of the cross section does not allow to separate these two terms. However, each of them have a distinct beam energy dependence that will be exploited in a second generation of experiments (see below).

A similar experiment performed on a deuterium target [7] provides the same combination of GPDs, but with different flavor weights. Results in figure 5 show values of $\operatorname{Im} C^{I}$ compatible with zero for all values of the momentum transfer $t$. Nevertheless, this allows to constrain in a modeldependent way the contribution of the angular momentum of quarks to the nucleon spin (Eq. 1). This is shown in the right plot of figure 5.

\subsection{Hall B program}

Hall B has a large wealth of data with several combinations of beam and target spin measurements. The large acceptance of the CLAS detector allows every observable to be measured in a much wider 

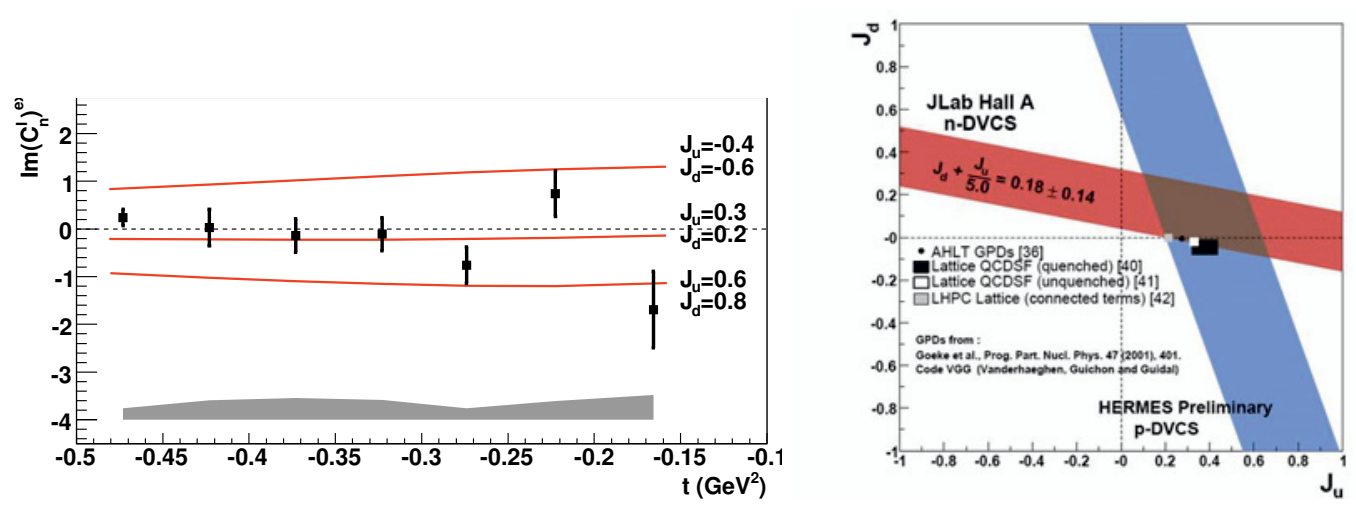

Figure 5. Left: linear combinations of GPDs $\operatorname{Im} C^{I}$ (Eq. 9) as a function of $t$ for the neutron. Right: Modeldependent extraction of the angular momentum of quarks $u$ and $d$ to the nucleon spin.

kinematic domain than is accessible in any of the other two experimental Halls. Extensively mapping three-dimensional distributions, such as GPDs, requires a vast kinematic survey that is best performed with a large acceptance detector such as CLAS and the future CLAS12. Multi-particle final state channels also benefit from the increased coverage and hermeticity of Hall B.

Figure 6 shows the amplitude $\alpha$ of the beam spin asymmetry:

$$
A=\frac{\vec{\sigma}-\stackrel{\leftarrow}{\sigma}}{\vec{\sigma}+\overleftarrow{\sigma}} \approx \frac{\alpha \sin \phi}{1+\beta \cos \phi}
$$

over a large kinematic domain. The comparison with simple models shows that at this moment not any single one of them is able to reproduce this observable all over the kinematic region accessible to experiment. The analysis of absolute cross sections over the same wide range is currently underway.

This wide set of data has been used to attempt GPD extraction directly by fitting GPD integrals at fixed $Q^{2}, x_{B}$ and $t$ [9]. Figure 7 shows the extraction performed using CLAS beam spin asymmetries $A_{L U}[8]$ and longitudinal target spin asymmetries $A_{U L}$ [10]. Both GPD combinations $\operatorname{Im} \mathcal{H}$ and $\operatorname{Im} \tilde{\mathcal{H}}$ are extracted with reasonable accuracy for some particular kinematics where these two observables exist simultaneously.

\subsection{New experiments under analysis}

Even though Jefferson Lab stopped its data taking 2 years ago in order to perform its energy upgrade, experiments that ran before the shutdown are still under analysis and will provide soon new results.

One of these experiments ran in Hall A with the goal of exploiting the beam energy dependence of the DVCS cross section in order to separate the BH.DVCS interference term from the pure DVCS ${ }^{2}$ contribution. Figure 8 shows projected results for the photon electroproduction cross section at the same kinematics: $Q^{2}, x_{B}$ and $t$, but two different beam energies: $E_{b}=6.0 \mathrm{GeV}$ and $E_{b}=3.6 \mathrm{GeV}$. At the same time, this experiment will perform the first $\mathrm{L} / \mathrm{T}$ separation of the $\pi^{0}$ electroproduction cross section, something that could not be done in previous experiments [11]. 


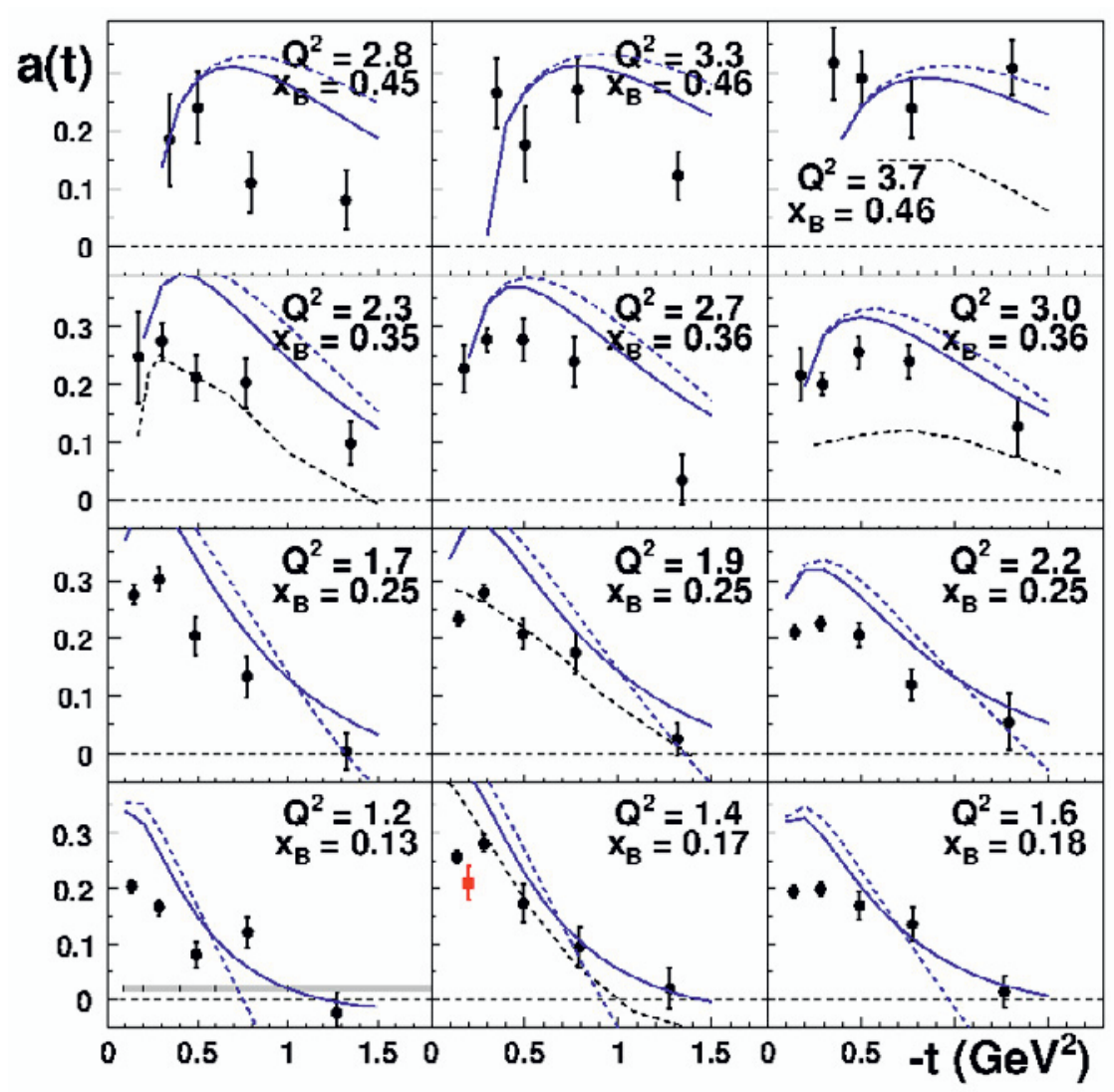

Figure 6. Amplitude of the DVCS beam spin asymmetry as a function of $t$ for several bins in $Q^{2}$ and $x_{B}$. Curves show the comparison with different models. See [8] for details.

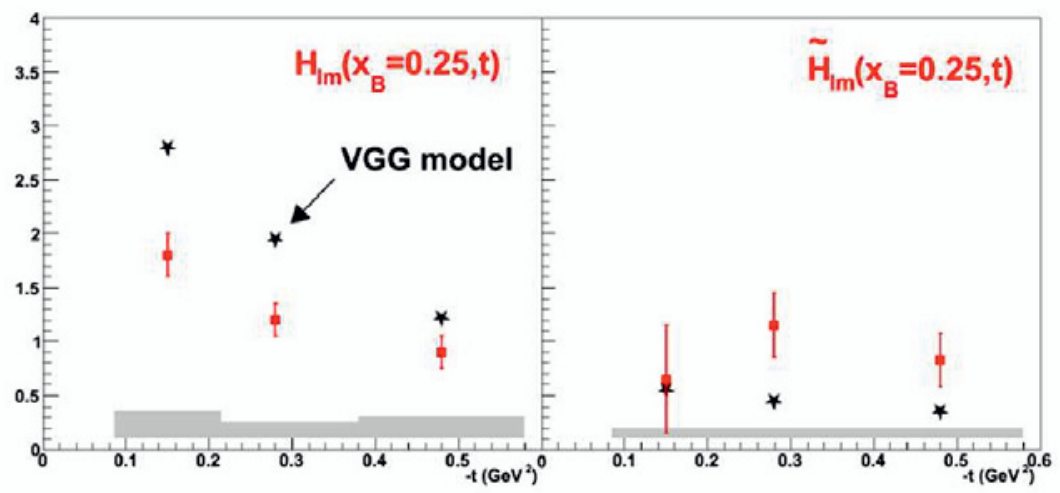

Figure 7. Extraction of the imaginary part of GPD integral $H$ (left) and $\tilde{H}$ (right) using the beam spin asymmetry and longitudinally target spin asymmetry from CLAS [9]. 

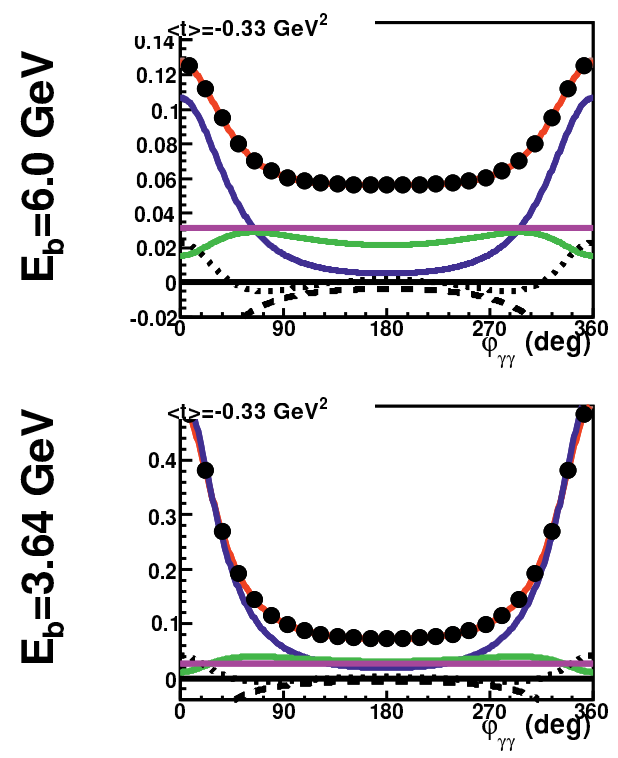

Figure 8. Projected results for the photon electroproduction cross section at $Q^{2}=1.5 \mathrm{GeV}^{2}$ and $x_{B}=0.36$ for two different beam energies: $E_{b}=6.0 \mathrm{GeV}$ (top) and $E_{b}=3.6 \mathrm{GeV}$ (bottom). The blue curves show the $\mathrm{BH}^{2}$ contribution. The beam energy changes the relative weight of $\mathrm{BH}$ with respect to DVCS, which allows the separation of each of these terms of the total cross section.

\section{$512 \mathrm{GeV}$ program}

Jefferson Lab is currently undergoing an upgrade of its beam energy from 6 to $12 \mathrm{GeV}$. The GPD program is at the heart of the scientific motivation driving this upgrade and an ambitious extensive program of measurements is already planned, starting as soon as Fall 2014. Figure 9 shows the $\left(Q^{2}\right.$, $x_{B}$ ) region that will be accessible with an $11 \mathrm{GeV}$ beam. Notice that even though the electron beam will be upgraded up to $12 \mathrm{GeV}$, only the new experimental Hall $\mathrm{D}$ will receive the maximum energy. All existing Halls (A, B and C) will be able to use a beam energy up to $11 \mathrm{GeV}$. Figure 9 illustrates the kinematic settings that will be covered by the first DVCS experiment with the upgraded beam [12] : E12-06-114 in Hall A. This experiment will perform a $Q^{2}$-dependence of the DVCS cross section at different values of $x_{B}$ with a lever-arm much higher than ever before. For comparison, the settings accessible with a $6 \mathrm{GeV}$ beam are also shown in black in the figure. This experiment is currently staging and will begin its data taking during Fall 2014.

Hall B has also an extensive program on GPD measurements using the upgraded beam. Figure 10 shows projected results for DVCS beam spin asymmetries in a wide kinematic range. Spanning the ranges $0.2<x_{B}<0.6$ and $1<Q^{2}<8 \mathrm{GeV}^{2}$ with a precision of a few percent for the asymmetries and systematic errors below $10 \%$ for the cross sections, the variations of the $t$-dependences across the $x_{B}$ range encode information on nucleon imaging of quark position in the transverse plane.

Finally, Hall $\mathrm{C}$ will also play a major role in the GPD program after the upgrade. The heavilyshielded detector setup in a highly-focusing magnetic spectrometer with large momentum reach, rigid connection to a sturdy pivot, well-reproducible magnetic properties, and access to the highestluminosity data, are essential factors for meaningful longitudinal-transverse cross section (L/T) separations. The anticipated excellent resolution and systematic understanding (less than $2 \%$ point-topoint) of the Hall-C spectrometer pair best address the experimental requirements for a program of precision L/T separations and ratios of exclusive cross sections with charged final states. Experiment E12-13-010 will perform a Rosenbluth separation of the DVCS cross section, using the same technique as the previous experiment E07-007 in Hall $\mathrm{A}$ at $6 \mathrm{GeV}$, but in the much larger kinematic range 


\section{DVCS measurements in Hall A/JLab}

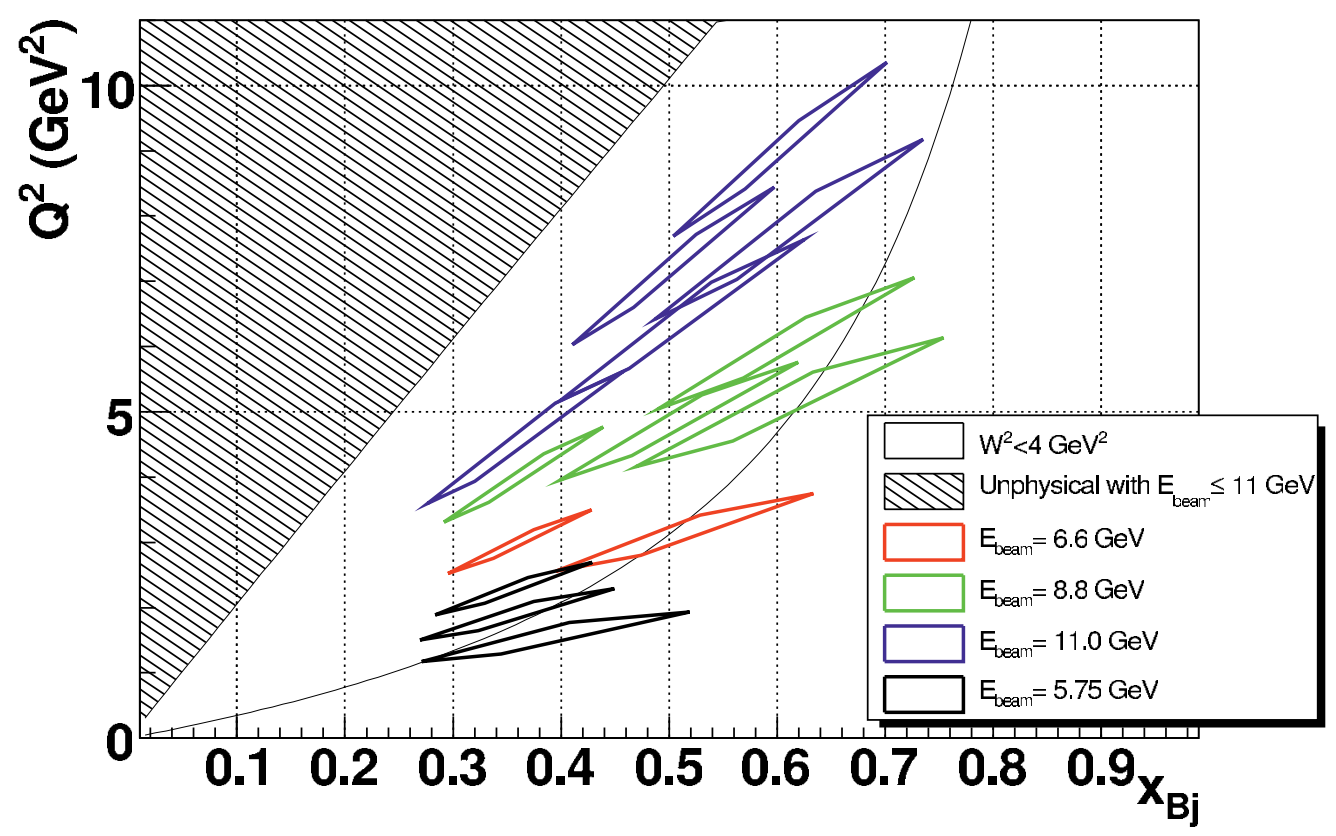

Figure 9. Kinematic region accessible with an $11 \mathrm{GeV}$ beam on a fixed target. The shaded top part represents the unphysical region $E_{b}>11 \mathrm{GeV}$ and the lower-right shade area corresponds to the region of invariant mass $W<2 \mathrm{GeV}$. For GPD measurements, in order to be away from the nucleon resonance region, an invariant mass of the system $W>2 \mathrm{GeV}$ is usually required.

available with an $11 \mathrm{GeV}$ beam. Figure 11 shows the kinematics settings covered by different beam energies (different colors).

\section{Summary}

GPDs are precious tools to probe the structure of the nucleon and DVCS is the golden channel to access GPDs. Jefferson Lab has a compelling experimental program aimed at studying the threedimensional structure of hadrons, in three different experimental Halls A, B and C. First precision data have already come out of the $6 \mathrm{GeV}$ program and results are very exciting. Indications of twist-2 dominance allow the interpretation of results in terms of GPDs to be in solid grounds. Thus, initial extraction of GPDs from data have been attempted. While still progress needs to be made on the theory side to make these extractions more and more reliable, these early results demonstrate the feasibility of the method and raise very interesting questions. Jefferson Lab at $12 \mathrm{GeV}$ will provide a huge wealth of high precision data in a much wider kinematic domain. A systematic and precision study of the 3D structure of nucleons will now begin.

\section{References}

[1] K.G. Wilson, Phys.Rev. D10, 2445 (1974) 


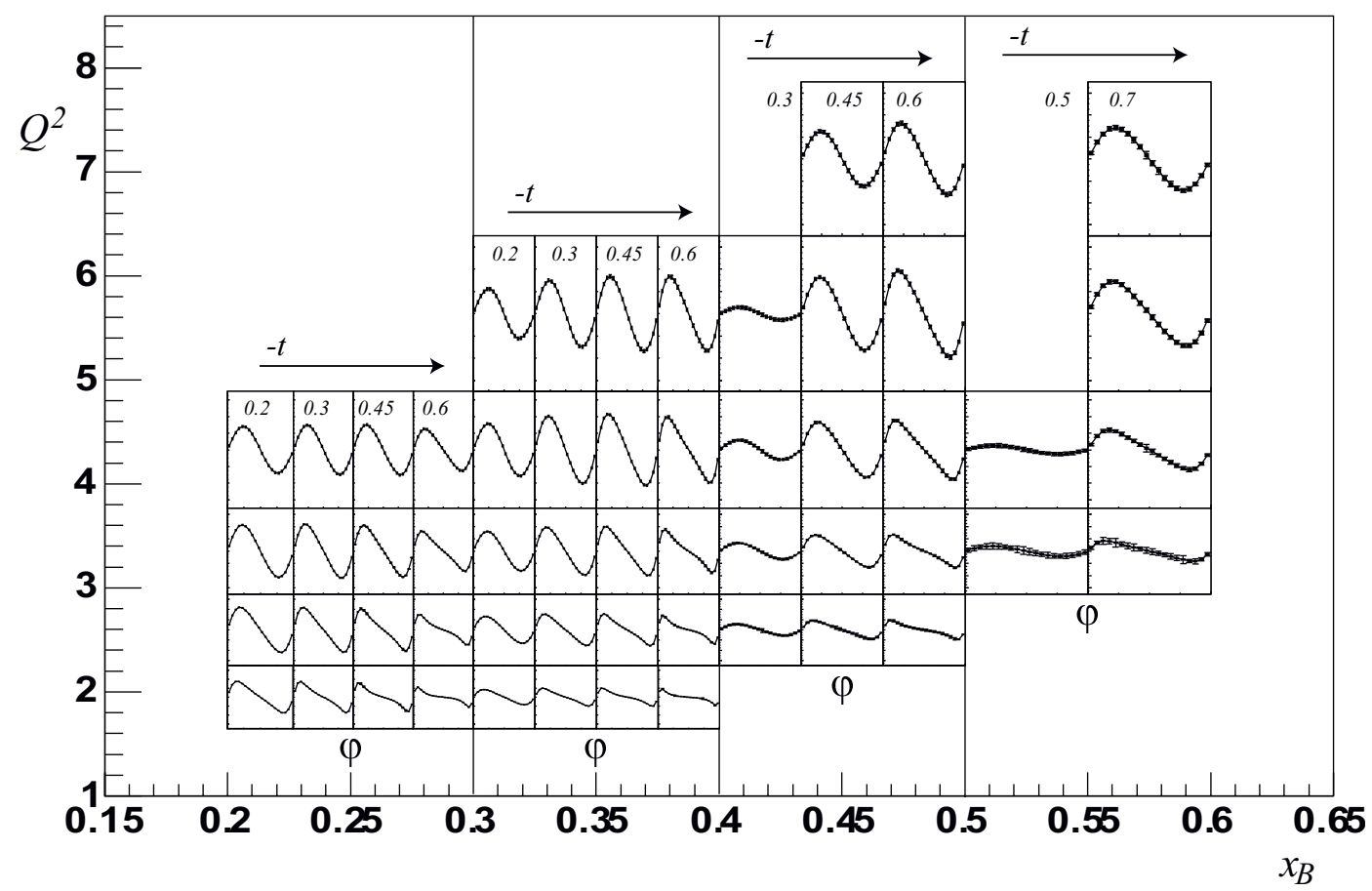

Figure 10. Projected results on DVCS beam spin asymmetries measurements in Hall B. Each plot represents one particular bin in $Q^{2}, x_{B}$ and $t$, as a function of the angle $\varphi$ between the leptonic and hadronic planes.

[2] B. Lucini, M. Panero, Phys.Rept. 526, 93 (2013), 1210. 4997

[3] D. Mueller, D. Robaschik, B. Geyer, F.M. Dittes, J. Horejsi, Fortschr. Phys. 42, 101 (1994), hep-ph/9812448

[4] A. Radyushkin, Phys.Lett. B380, 417 (1996), hep-ph/9604317

[5] X.D. Ji, Phys. Rev. Lett. 78, 610 (1997), hep-ph/9603249

[6] C. Muñoz Camacho et al., Phys.Rev.Lett. 97, 262002 (2006), nucl-ex/0607029

[7] M. Mazouz et al., Phys.Rev.Lett. 99, 242501 (2007), 0709.0450

[8] F. Girod et al. (CLAS Collaboration), Phys.Rev.Lett. 100, 162002 (2008), 0711.4805

[9] M. Guidal, Phys.Lett. B689, 156 (2010), 1003.0307

[10] S. Chen, H. Avakian, V. Burkert, P. Eugenio (CLAS) (2006), hep-ex/0605012

[11] E. Fuchey et al., Phys.Rev. C83, 025201 (2011), 1003.2938

[12] J. Roche, C. E. Hyde-Wright, B. Michel and C. Muñoz Camacho (spokespersons), PR12-06-114, approved by PAC30 (2006), nucl-ex/0609015 


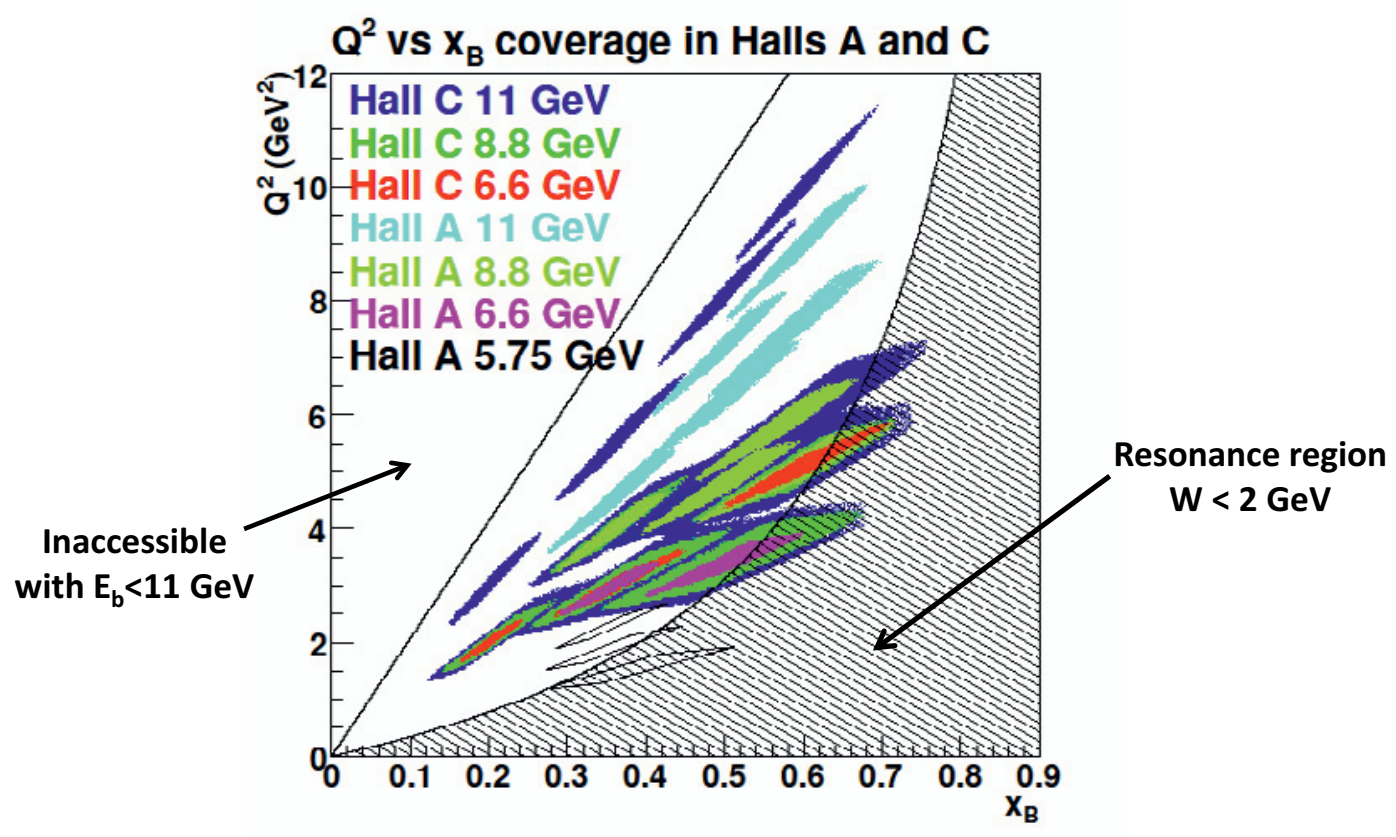

Figure 11. Combined kinematic coverage in $Q^{2}$ and $x_{B}$ between Hall A and Hall C DVCS experiments. Different colors at the same setting indicate that the cross section will be measured at different incident beam energies, which allows the separation of the DVCS ${ }^{2}$ contribution from the BH.DVCS interference term. 